\title{
Estratégias de enfrentamento utilizadas por cuidadores de crianças com deficiência
}

\author{
Coping strategies used by caregivers of children with deficiency
}

\section{Estrategias de afrontamiento utilizadas por los cuidadores de los niños con discapacidad}

Bárbara Bernadete de Oliveira Brito ${ }^{1}$, Francisco Orlando Rafael Freitas ${ }^{1,2}$, Charlene de Oliveira Pereira1, André Luiz Dantas Bezerra², Paula Christianne Gomes Gouveia Souto Maia², Everson Vagner de Lucena Santos ${ }^{1,2}$, Petrônio Souto Gouveia Filho1, Gildenia Pinto dos Santos Trigueiro', Miguel Aquila Toledo1, Vandezita Dantas de Medeiros Mazzaro1, Larissa de Araújo Batista Suárez ${ }^{2,3,4}$, Fabiana Casusa de Oliveira ${ }^{5}$, Milena Nunes Alves de Sousa1,2*.

\section{RESUMO}

Objetivo: Avaliar estratégias de enfrentamento adotadas pelos cuidadores de crianças com deficiência no município de Patos-PB. Métodos: Pesquisa de campo, descritiva, transversal, com abordagem quantitativa, efetuada com 18 cuidadores de crianças com deficiência atendidas no Centro de Equoterapia. Aplicou-se o "Inventário de Estratégias de Coping". A análise ocorreu descritivamente, mediante estatísticas: média, desvio padrão e mediana, frequências absolutas e percentuais. Resultados: As estratégias de coping mais utilizadas foram resolução de problemas (84\%), reavaliação positiva (77\%) e suporte social $(76,9 \%)$. A menos utilizada foi fuga-esquiva (20\%). Conclusão: Destacou-se a estratégia de enfrentamento mais conhecida como resolução de problemas, entretanto, fuga-esquiva foi a menos adotada. Explorar mais sobre as estratégias de enfrentamento utilizadas pelos cuidadores irá possibilitar compreender melhor sobre os problemas diários enfrentados, além de contribuir para um manejo eficaz e amparar no cuidado com as crianças com deficiência.

Palavras-Chave: Estratégias de Enfrentamento, Cuidadores, Deficiência.

\begin{abstract}
Objective: To evaluate coping strategies adopted by caregivers of children with deficiency in Patos city. Methods: Transversal research, quantitative, performed with 18 caregivers of children with disabilities served in Equestrian Center. Applied the "Coping Strategies Inventory". The analysis was descriptive by means of statistics: mean, standard deviation and median, absolute and percentage frequencies. Results: The most used coping strategies were problem solving $(84 \%)$, positive reevaluation $(77 \%)$ and social support $(76.9 \%)$. The least used was fugue-avoidance (20\%). Conclusion: It was highlighted the coping strategy best known as troubleshooting; however, escape-avoidance was the least adopted. Exploring more about the coping strategies used by caregivers will make it possible to better understand the daily problems faced, besides contributing to an effective management and support in caring for children with disabilities.
\end{abstract}

Keywords: Coping Strategies. Caregivers. Deficiency.

\section{RESUMEN}

\footnotetext{
${ }^{1}$ Centro Universitário de Patos UNIFIP, Patos-PB, Brasil. *E-mail: minualsa@hotmail.com

2 Faculdade Vale do Pajeú, São José do Egito-PE, Brasil.

${ }^{3}$ Universidade Católica de Pernambuco, Recife-PE, Brasil.

${ }^{4}$ Faculdade São Francisco da Paraíba, Cajazeiras-PB, Brasil.

${ }^{5}$ Secretaria de Saúde de Itaporanga, Itaporanga-PB, Brasil.
} 
Objetivo: evaluar el afrontamiento estrategias adoptadas por los cuidadores de los niños discapacitados en la ciudad de Patos-PB. Métodos: Investigación de campo, descriptiva, transversal, con enfoque cuantitativo, realizada con 18 cuidadores de niños con discapacidad sirvió en el centro de Equinoterapia. Aplicó el "inventario de estrategias de afrontamiento" y el "cuestionario para la familia". El análisis se produjo a través de estadística: descriptiva media, desviación estándar y mediana de frecuencias absolutas y porcentajes. Resultados: Las estrategias de afrontamiento más utilizadas fueron la resolución de problemas (84\%), la revalorización positiva $(77 \%)$ y apoyo social $(76,9 \%)$. El menos utilizado fue la prevención de escape (20\%). Conclusión: Estrategia de afrontamiento conocido como resolución de problemas, sin embargo, fue el menos adoptado escape-evitación. Descubra más sobre afrontamiento estrategias utilizadas por los cuidadores permitirá una mejor comprensión sobre los problemas diarios, además de contribuir a una gestión eficaz y la ayuda en el cuidado de los niños con discapacidades.

Palabras clave: Afrontamiento Estrategias, Cuidadores, Discapacidad.

\section{INTRODUÇÃO}

Crianças que apresentam algum tipo de deficiência expressam distúrbios neuro-sensório-motores que restringem sua atividade na sociedade (PAVÃO S, SILVA F e ROCHA N, 2011). Neste grupo, o papel da família, amigos e cuidadores é essencial para o bem-estar do infante. Devem sempre manter a ligação amorosa preservada, bem como seus ensinamentos, pois são nos cuidadores que as crianças têm seu referencial (SILVEIRA A, NEVES ET e PAULA CC, 2013). Filhos com limitações demandam cuidados diferenciados, como também tratamento e maior dedicação. A maior dedicação ao cuidado foi relatada pelos cuidadores durante período de nascimento do filho, como também momentos de mudança, como acesso à escola, entrada na adolescência (RIBEIRO MFM, et al, 2016).

Toda família está sujeita ao estresse quando se trata do cuidado de uma criança e seu desenvolvimento adequado. Naquelas com crianças deficientes ou com doença crônica, muitas vezes os níveis de estresse podem ter um peso maior. É importante que haja estratégias de enfrentamento que possam ser utilizadas por todos os familiares e cuidadores, para que exerçam influência no cuidado dessas crianças e suas limitações (COLETTO M e CÂMARA S, 2009).Conforme os autores, a estratégia de coping é uma resposta ao momento de estresse, com a finalidade de manter o controle pessoal, de certa forma como lidar o evento estressor. Pode estar focado no problema ou na emoção. Ou seja, uma resposta ao ato de estresse quando ligado ao problema ou uma resposta emocional ao episódio estressante.

Cuidadores que buscam estratégias de enfrentamento desenvolvem melhores resultados, pois buscam por alternativas que aliviem as dificuldades, planejem tratamentos, procuram por melhor controle da doença. O enfrentamento do problema, por meio de táticas de reavaliação positiva, permite benefícios nos resultados e máxima eficiência do cuidado (COLETTO M e CÂMARA S, 2009). Cabe, portanto, aos cuidadores dessas crianças, buscar diminuir essas restrições, aumentando a função desse grupo na sociedade e no dia a dia da mesma. É importante atribuir aos cuidadores orientações e receberem também uma assistência para que obtenham eficácia na busca de aumentar a funcionalidade das crianças com deficiência, no quesito tanto de autocuidado quanto na sua inserção social (PAVÃO S, SILVA F e ROCHA N, 2011).

Assim, objetivou-se avaliar as estratégias de enfrentamento adotadas pelos cuidadores de crianças com deficiência no município de Patos-PB. A temática apresenta relevância tendo em vista que o grupo enfrenta dificuldades diárias.

\section{MÉTODOS}

Trata-se de uma pesquisa de campo, descritiva, transversal, com abordagem quantitativa. O estudo foi realizado no em um centro de equoterapia do município de Patos, localizado no sertão paraibano. O lugar 
atende crianças de 2 a 13 anos de idade, com quadros clínicos de Paralisia Cerebral, Síndrome de Down e Transtornos do Espectro Autista.

A coleta de dados foi realizada pela pesquisadora responsável, a partir da entrega e esclarecimento do questionário validado em português intitulado de "Inventário de Estratégias de Coping" (IEC) de Folkman e Lazarus (1984), traduzido no Brasil por Savóia M, Santana P e Mejias N (1996). Depois de esclarecidos os propósitos da pesquisa, entre os sujeitos que manifestam interesse em participar do estudo assinando 0 Termo de Consentimento Livre e Esclarecido, foi dado 30 minutos para responder ao instrumento de coleta. Constituíram a amostra, 18 indivíduos (78,3\% do universo).

O IEC é composto por 66 itens que abordam ações e pensamentos utilizados em situações estressantes. Portanto, avalia variáveis da personalidade e a escolha de estratégias em situações estressantes, que foram divididos em oito fatores classificatórios: aceitação de responsabilidade, afastamento, autocontrole, confronto, fuga e esquiva, reavaliação positiva, resolução de problemas e suporte social. Avaliado por meio de uma escala de 0 (não utiliza) a 3 pontos (utiliza em grande quantidade). Para melhor compreensão das estratégias, o Quadro 1 descreve seu significado (LAZARUS RS e FOLKMAN S, 1984).

Quadro 1 - Significado das Estatégias de Coping.

\begin{tabular}{|l|l|}
\hline Estratégias de Coping & Significado \\
\hline $\begin{array}{l}\text { Aceitação } \\
\text { responsabilidade }\end{array}$ & $\begin{array}{l}\text { Entendimento do papel na conjuntura vivida e busca por tentar solucionar o } \\
\text { problema }\end{array}$ \\
\hline Afastamento & $\begin{array}{l}\text { Esforços cognitivos de abnegação e desprendimento, bem como minimização } \\
\text { da conjuntura vivida }\end{array}$ \\
\hline Autocontrole & Empenho em regular os próprios sentimentos, vivencias e ações \\
\hline Confronto & $\begin{array}{l}\text { Esforços brutos para alterar a situação vivenciada, podendo ser hostil e } \\
\text { apresentar grau de risco }\end{array}$ \\
\hline Fuga e esquiva & Estratégias para evitar ou escapar do problema \\
\hline Reavaliação positiva & $\begin{array}{l}\text { Reconfiguração da situação vivenciada, na tentativa de ressignificar o } \\
\text { problema para uma percepção mais positiva, podendo apresentar a atitude } \\
\text { religiosa }\end{array}$ \\
\hline Resolução de problemas & Focalização no problema para alterá-lo ou solucioná-lo \\
\hline Suporte social & Busca por informação, apoio tangível e emocional \\
\hline
\end{tabular}

Fonte: Próprios autores, 2019. Adaptado de Lazarus RS e Folkman S (1984).

A coleta de dados foi realizada no período de fevereiro a maio após aprovação pelo Comitê de Ética em Pesquisa das Faculdades Integradas de Patos - CAAE: 79819917.0.0000.5181/Protocolo de Aprovação: 2.438.333/2017. Os dados foram calculados no Statistical Package for the Social Sciences, versão 23. A análise ocorreu descritivamente mediante estatísticas: média, desvio padrão e mediana.

\section{RESULTADOS}

Na Tabela 1 apresentam-se os resultados da pesquisa de coping de todas as respostas dos itens dos fatores. Desta tabela verifica-se com exceção do fator fuga-esquiva, em que 55,6\% não haviam utilizado; nos demais fatores a maioria fez uso de estratégia, sendo mais elevado nos fatores: não usou na resolução de problema $(14,8 \%)$, não usou na reavaliação positiva $(23,0)$ e não usou no suporte social $(23,1 \%)$; o percentual que fez uso de grande quantidade das estratégias de enfrentamento, variou de $9,0 \%$ a $27,8 \%$, sendo mais elevados nos fatores resolução de problema $(27,8 \%)$, reavaliação positiva $(27,8 \%)$ e suporte social $(23,1 \%)$; as frequências dos que fizeram uso bastante variaram de $8,3 \%$ a $31,5 \%$, sendo mais elevadas na resolução de problemas $(31,5)$, reavaliação positiva $(30,2 \%)$ e suporte social $(28,7 \%)$; na categoria um pouco o maior percentual foi no autocontrole $(34,1 \%)$, contrariamente o menor foi quanto a reavaliação positiva $(19,0 \%)$, com variações entre $25,0 \%$ a $28,7 \%$ nos demais fatores. 
Tabela 1 - Frequência de utilização das estratégias de coping no total de respostas.

\begin{tabular}{|c|c|c|c|c|c|c|c|c|c|}
\hline \multirow{3}{*}{ Fatores do coping } & \multicolumn{8}{|c|}{ Uso de estratégia } & \multirow{3}{*}{ Total } \\
\hline & \multicolumn{2}{|c|}{ Não usei } & \multicolumn{2}{|c|}{ Um pouco } & \multicolumn{2}{|c|}{ Bastante } & \multicolumn{2}{|c|}{$\begin{array}{c}\text { Grande } \\
\text { quantidade }\end{array}$} & \\
\hline & $\mathbf{n}$ & $\%$ & $\mathbf{n}$ & $\%$ & $\mathbf{n}$ & $\%$ & $\mathbf{n}$ & $\%$ & \\
\hline \multirow{2}{*}{$\begin{array}{l}\text { Aceitação/ } \\
\text { responsabilidade }\end{array}$} & & & & & & & 1 & & \multirow[t]{2}{*}{72} \\
\hline & 33 & 45,8 & 18 & 25,0 & 11 & 15,3 & 0 & 13,9 & \\
\hline \multirow{2}{*}{ Afastamento } & & & & & & & 1 & & \multirow[t]{2}{*}{108} \\
\hline & 41 & 38,0 & 31 & 28,7 & 23 & 21,3 & 3 & 12,0 & \\
\hline \multirow{2}{*}{ Autocontrole } & & & & & & & 1 & & \multirow[t]{2}{*}{126} \\
\hline & 41 & 32,5 & 43 & 34,1 & 23 & 18,3 & 9 & 15,1 & \\
\hline \multirow[t]{2}{*}{ Confronto } & 51 & & 28 & 259 & 17 & & $\begin{array}{l}1 \\
?\end{array}$ & 111 & 108 \\
\hline & 31 & $4 l, 2$ & 28 & $\angle 0,9$ & 17 & 15,1 & $\begin{array}{l}2 \\
1\end{array}$ & 11,1 & \multirow{2}{*}{144} \\
\hline Fuga-esquiva & 80 & 55,6 & 39 & 27,1 & 12 & 8,3 & 3 & 9,0 & \\
\hline \multirow{2}{*}{ Reavaliação positiva } & & & & & & & 3 & & \multirow[t]{2}{*}{126} \\
\hline & 29 & 23,0 & 24 & 19,0 & 38 & 30,2 & 5 & 27,8 & \\
\hline \multirow{2}{*}{$\begin{array}{l}\text { Resolução } \\
\text { problema }\end{array}$} & & & & & & & 3 & & \multirow[t]{2}{*}{108} \\
\hline & 16 & 14,8 & 28 & 25,9 & 34 & 31,5 & 0 & 27,8 & \\
\hline \multirow{2}{*}{ Suporte social } & & & & & & & 2 & & \multirow[t]{2}{*}{108} \\
\hline & 25 & 23,1 & 27 & 25,0 & 31 & 28,7 & 5 & 23,1 & \\
\hline
\end{tabular}

Fonte: Próprios autores, 2019.

\section{DISCUSSÃO}

O nascimento de um bebê na família atinge todos os membros, sendo visto muitas vezes como um dos momentos mais marcantes e importantes para a vida da mãe. Contudo, ao descobrir provável deficiência do filho ou alguma condição clínica mais severa, em geral, a primeira reação das mães é de negação e preocupação de correr risco de morte. Outros sentimentos envolvidos nesse primeiro momento envolvem a surpresa, insegurança e preocupação. É nesse momento que os cuidadores já buscam por ajuda com profissionais de saúde para entender melhor a gravidade e desenvolvimento da criança e dessa forma buscar a diminuição do sofrimento e a aceitação (SILVA CX, et al., 2010; FIRMINO CDB e SOUSA MNA, 2013).

Sentimentos de medo e incapacidade de cuidar daquela criança com deficiência ou com uma doença mais severa também são comuns. Ainda, o diagnóstico do filho e/ou um ente querido com alguma limitação gera sensação de choque, raiva, tristeza, dor e sentimento de impotência. Esta realidade é passível de estresse, depressão e sintomas de ansiedade (IACONO T, et al., 2016; ISESELO MK, KAJULA L, YAHYA-MALIMA, 2016; NIPP RD, et al., 2016; TILAHUN D, et al., 2016).

Pais e cuidadores podem vivenciar tais sentimentos por toda a vida e ainda adoecerem, sendo oportuno buscar tranquilidade e afastar emoções negativas, adotando táticas para lidar com o estresse e amenizar tais sentimentos (SANTOS MA e PEREIRA-MARTINSMLPL, 2016). Apenas com o passar do tempo, vão desenvolvendo habilidades e estratégias de enfrentamento do estresse inicial (FIRMINO CDB e SOUSA MNA, 2013; PINTANEL AC, GOMES GC e XAVIER DM, 2013).

Assim, buscando identificar as estratégias de coping, foi possível verificar que a estratégia mais utilizada foi resolução de problemas, seguida de reavaliação positiva e suporte social. A menos utilizada foi fugaesquiva. Pelas características das estratégias adotadas é sugestivo que os cuidadores são resilientes. Resiliência trata de um conjunto de situações já vivenciadas e que proporcionaram aprendizados, sendo possível a superação das ocasiões que causaram angústia. Além disso, proporciona crescimento pessoal e melhor desenvolvimento (YUNES MAM, FERNANDES G, WESCHENFELDER GV, 2018).

Estudo realizado com familiares de pacientes oncológicos, utilizando o instrumento de coping constatou que todos os participantes fizeram uso das estratégias de enfrentamento, sendo o fator com maior índice o 
de reavaliação positiva, seguido de suporte social, ambos também utilizados com frequência nesse estudo. Semelhante a esta pesquisa com cuidadores de crianças com deficiência, a estratégia de enfrentamento fugaesquiva apresentou a menor média entre os familiares de pacientes oncológicos (FETSCH CFM, et al., 2016).

Em investigação com familiares diante um membro da família hospitalizado e utilização de estratégias de coping, as medidas mais utilizadas foram convergentes com o estudo: reavaliação positiva, o suporte social e a resolução de problemas. Sendo as de menor frequência também semelhante: autocontrole, fuga-esquiva, aceitação/responsabilidade, confronto e afastamento. A menor frequência na utilização dessas estratégias não exclui o seu uso e sua importância, mas indica que os familiares encaram as mesmas como estratégias defensivas, com maior envolvimento emocional, podendo gerar má conformação pessoal (SANTOS QN, 2013).

Outra pesquisa com pais de crianças com doenças crônicas destacou-se o uso da estratégia de enfrentamento reavaliação positiva, sugerindo que os cuidadores possuem expectativas positivas e esperança de melhora do quadro da criança. A reavaliação positiva foi a segunda mais utilizada, seguida por suporte social e resolução de problemas (COLETTO M e CÂMARA S, 2009). Essas estratégias em uso assemelham-se a este estudo.

Sobre a resolução de problemas, categoria mais utilizada na pesquisa, é oportuno enfatizar que a mesma contempla a realização de um plano de ação e busca cumpri-lo para modificar o estado de saúde do filho, nesta abordagem, as deficiências. Assim, indica que os cuidadores sabiam o que deveria ser feito e redobraram seus esforços para alcançar o necessário (LAZARUS RS e FOLKMAN S, 1984; DAMIÃO EBC, et al., 2009). Em estudo com pais de crianças portadoras de deficiência intelectual, a estratégia resolução de problemas, a busca do suporte social, bem como reavaliação positiva (fé e religião), também foram estratégias utilizadas para o enfrentamento dos desafios do cuidado. As estratégias relacionadas com a emoção igualmente foram pouco empregadas. Na resolução de problemas, as estratégias foram vistas como importantes em situações em que se pode mudar a situação, uma busca para melhora da qualidade de vida e controle de ansiedade e depressão (SANTOS MA e PEREIRA-MARTINSMLPL, 2016).

O fator reavaliação positivafoi utilizada de modo igualmente importante neste estudo. $O$ uso frequente dessa estratégia indica que os pais e cuidadores, apesar de todas as dificuldades, ainda assim apresentam esperança e pensamento positivo de mudança da situação. Busca controle das emoções negativas, como forma de modificação a partir da condição conflitante (COLETTO M e CÂMARA S, 2009). No presente estudo, foi possível verificar que dentre os itens deste fator, os mais utilizados foram os itens rezei, seguido de redescobri o que é importante na vida. Tais elementos assemelharam-se as dos familiares de pacientes oncológicos (FETSCH CFM, et al., 2016).

Por conseguinte, no presente estudo, suporte social foi uma estratégia que também teve representativa quanto ao uso, bem como na pesquisa sobre "Estratégias de Coping entre Familiares de Pacientes Oncológicos" (FETSCH CFM, et al., 2016). A busca por ajuda profissional e aceitação da simpatia e compreensão das pessoas foram bem enfatizadas entre os cuidadores de deficientes. Cabe ressaltar que o uso da estratégia de enfrentamento suporte social é fundamental, pois provê a rede de apoio para encarar situações de estresse (MACEDO CM, et al., 2018).

Estudo sobre "coping e estresse familiar e enfrentamento na perspectiva do transtorno do espectro do autismo", também enfatizaram a necessidade de uma rede de suporte, pois visualizaram que muitas são as dificuldades relativas ao cuidado de uma criança com o transtorno do espectro autístico (CARVALHO-FILHA FSS, et al., 2018). A estratégia de fuga-esquiva, como relatado, foi a menos utilizada. Essa estratégia possui respostas que tem como propósito prorrogar modificações da situação estressante. Os cuidadores passam a aceitar o quadro e consequências da doença, como se já fizessem parte da sua rotina. O menor uso dessa estratégia relaciona-se ao sentimento de aceitação. Os pais buscam por uma postura mais ativa para a resolução de problemas e modificação do quadro do filho (COLETTO M e CÂMARA S, 2009).

O confronto satisfaz às estratégias em que cuidadores apresentam atitudes ofensivas para a resolução da situação, buscando um culpado, mostrando estar com raiva, extravasado os sentimentos. Tais estratégias 
foram utilizadas em menor quantidade pelos cuidadores (49\%). Opondo-se as estratégias de confronto têmse as de afastamento. Nesta, busca-se evitar o confronto e mudar pouco a situação causadora de problema, permanecendo em defensiva. Seu uso também não foi muito frequente (59\%), tendo em vista que os pais de crianças com deficiência estão em busca de melhor estado de saúde e bom prognóstico no futuro, evitando aceitar as limitações (DAMIÃO EBC, et al., 2009).

O autocontrole trata justamente de buscar conter as emoções e não agir por impulso em situações conflitantes. Uma das estratégias dessa categoria, bastante utilizada pelos pais foi analisar mentalmente 0 que fazer e o que dizer, $88,9 \%$ dos pais em algum momento fizeram uso dessa estratégia. A aceitação de responsabilidade foi adotada por $67 \%$ dos cuidadores e mesma enfatiza o saber lidar com a ação estressante, que pode até mesmo estimular a busca de enfrentar tal situação de outra forma, manifestando autonomia (DAMIÃO EBC, et al., 2009). Por conseguinte, com o avançar da idade do filho, as estratégias de enfrentamento tornam-se mais utilizadas, deixando os cuidadores mais seguros e facilitando a retomada dos planos de vida. $O$ amor transmitido ao filho, aceitar a situação e experiências vividas são essenciais para adaptação (RIBEIRO MFM, et al., 2016).

\section{CONCLUSÃO}

Os achados indicam que as estratégias de enfrentamento mais utilizadas foram as de resolução de problemas, reavaliação positiva e suporte social. Contrariamente, a fuga-esquiva foi a menos adotada. Explorar mais sobre as estratégias de enfrentamento utilizadas pelos cuidadores irá possibilitar compreender melhor sobre os problemas diários enfrentados, além de contribuir para um manejo eficaz e amparar no cuidado com as crianças com deficiência.

\section{REFERÊNCIAS}

1. PAVÃO S, SILVA F, ROCHA N. Efeito da orientação domiciliar no desempenho funcional de crianças com necessidades especiais. Motricidade, 2011;7(1):21-9.

2. SILVEIRA A, NEVES ET, PAULA CC. Cuidado familial das crianças com necessidades especiais de saúde: um processo (sobre)natural e de (super)proteção. Texto Contexto Enferm., 2013;22(4): 1106-14.

3. RIBEIRO MFM, et al. Paralisia cerebral: faixa etária e gravidade do comprometimento do filho modificam o estresse e o enfrentamento materno. Ciênc. Saúde Colet., 2016; 21(10), 3203-12.

4. COLETTO M, CÂMARA S. Estratégias de coping e percepção da doença em pais de crianças com doença crónica: o contexto do cuidador. Diversitas: Perspectivas en Psicología., 2009;5(1):97-110.

5. LAZARUS RS, FOLKMAN S. Stress, appraisal, and coping. New York: Springer; 1984.

6. SAVÓIA M, SANTANA P, MEJIAS N. Adaptação do inventário de Estratégias de Coping de Folkman e Lazarus para o português. Psicologia USP, 1996;7(1-2):183-201.

7. SILVA CX, et al.. Criança com paralisia cerebral: qual o impacto na vida do cuidador? Rev. da Rede de Enf. do Nordeste., 2010;11:204-214.

8. FIRMINO CDB, SOUSA MNA. Sentimentos e vivências de familiares em frente ao diagnóstico de câncer na criança. Rev. Bras. Pesq. Saúde, 2013;15(2):6-12.

9. PINTANEL AC, GOMES GC, XAVIER DM. Mães de crianças com deficiência visual: dificuldades e facilidades enfrentadas no cuidado. Rev. Gaúch. Enferm., 2013;34(2):86-92.

10. ISESELO MK, KAJULA L, YAHYA-MALIMA KI. The psychosocial problems of families caring for relatives with mental illnesses and their coping strategies: a qualitative urban based study in Dar es Salaam, Tanzania. BMC Psychiatry, 2016; 16:146.

11. NIPP RD, et al. Factors associated with depression and anxiety symptoms in family caregivers of patients with incurable cancer. Ann Oncol., 2016;27(8):1607-12.

12. TILAHUN D, et al. Stigma, explanatory models and unmet needs of caregivers of children with developmental disorders in a lowincome African country: a cross-sectional facility-based survey. BMC Health Serv Res., 2016;16: 152.

13. IACONO T, et al. Family caring of older adults with intellectual disability and coping according to loci of responsibility. Res DevDisabil., 2016; 57: 170-80.

14. SANTOS MA, PEREIRA-MARTINS MLPL. Estratégias de enfrentamento adotadas por pais de crianças com deficiência intelectual. Ciênc. Saúde Colet., 2016;21(10):3233-44.

15. YUNES MAM, FERNANDES G, WESCHENFELDER GV. Intervenções psicoeducacionais positivas para promoção de resiliência: o profissional da educação como tutor de desenvolvimento. Educação, 2018; 41(1): 83-92.

16. FETSCH CFM, et al. Estratégias de Coping Entre Familiares de Pacientes Oncológicos. Rev. Bras. Cancerol., 2016;62(1):17-25.

17. SANTOS QN. Estratégia de Enfrentamento (Coping) da Família Frente a um Membro Familiar Hospitalizado. Uma Revisão de Literatura Brasileira. Mudanças, Psicol. Saúde, 2013;21(2):40-7.

18. DAMIÃO EBC, et al. Inventário de estratégias de enfrentamento: um referencial teórico. Rev. Esc. Enferm. USP, 2009; 43(spe2): 1199-203.

19. MACEDO CM, et al. Coping strategies for domestic violence against pregnant female adolescents: integrative review. RevBrasEnferm., 2018;71(supl1):693-9.

20. CARVALHO-FILHA FSS, et al. Coping e estresse familiar e enfrentamento na perspectiva do transtorno do espectro do autismo. Rev. Cient. Sena Aires,2018; 7(1):23-30. 